\title{
Nuevas políticas públicas y sistemas de governance en los distritos industriales italianos
}

\section{New public policies and governance systems in the Italian industrial districts}

\author{
RODRIGO REYNALDO-CARMONA*
}

\begin{abstract}
In this paper the experience of the Italian industrial district as an object of public policy is analysed. Firstly, we describe the main theoretical and structural characteristics that distinguish this kind of productive organization and its dynamics over time. Secondly, we examine the district's experience taking into account the Italian industrial policies at national and regional levels. We also address the institutional architecture, as well as the different agents and strata that constitute the decision making process (governance) of the industrial policy at the territorial level. Finally, we reach some possible conclusions about the importance of the industrial district and its implications in public policy and local development.
\end{abstract}

Keywords: industrial district, public policies, governance, territory.

\section{Resumen}

El presente artículo analiza la experiencia del distrito industrial italiano como objeto de política pública. En primer lugar, se describen las principales características teóricas y estructurales que distinguen a este tipo de organización productiva y su dinámica en el tiempo. En segundo término, se examina la experiencia del distrito contemplando la política industrial italiana en el plano nacional y regional. Posteriormente se analiza la arquitectura institucional y los distintos actores y niveles que conforman el proceso decisional (governance) de la política industrial en el plano territorial. Por último, se hacen algunas conclusiones tentativas sobre la importancia del distrito industrial y sus implicaciones en el ámbito de las políticas públicas y del desarrollo local.

Palabras clave: distrito industrial, políticas públicas, governance, territorio.

* Universidad Nacional de General Sarmiento, Argentina. Correo-e: rcarmona@ ungs.edu.ar. 


\section{Introducción}

En las últimas décadas los distritos industriales son objeto de debate científico y estudio por parte de distintos observadores en el ámbito mundial. Su importancia se vincula con la aparición de nuevas formas productivas más flexibles que destacan el rol de las firmas de menor envergadura y sus lugares de origen en la economía real. Estos sistemas, en el marco actual de globalización y competencia creciente, afrontan distintos desafíos en lo que se refiere a la innovación tecnológica, los cambios institucionales y su inserción especializada en redes internacionales de producción.

En términos generales, los distritos industriales representan una modalidad de organización de la producción que tiene a las pequeñas y medianas empresas (PyMEs) en la dimensión territorial como elementos centrales. Un primer aspecto que se considera desde el punto de vista teórico es el hecho de que una concentración geográfica asociada a un tipo de especialización sectorial genera economías externas que pueden aprovechar las firmas (Marshall, 1920). Estas externalidades pueden ser de tipo pecuniario cuando la cercanía entre empresas genera rendimientos crecientes en la industria, tanto porque reduce los costos de transporte ligados a los procesos de integración vertical y horizontal de la producción, como porque se obtienen beneficios de la utilización conjunta de algunos recursos localizados en relación con la creación de un mercado de trabajo especializado, oferta de materias primas y maquinaria, infraestructura y servicios especializados (Krugman, 1991). Las externalidades tecnológicas se desarrollan cuando la proximidad geográfica favorece la distribución de conocimiento crítico y la difusión de innovaciones, creando un contexto de interacciones y elevada conectividad que reduce los costos de transacción entre empresas, fundamentalmente en presencia de tecnologías fluidas (Antonelli, 1999). Por otra parte, en algunos casos las empresas localizadas en los distritos industriales pueden disfrutar también de otros tipos de externalidades, como las de tipo reputacional o de naturaleza más institucional, ligadas a intercambio de confianza, al buen funcionamiento de mercados internos, relaciones de reciprocidad y mayor articulación entre las demandas individuales y las instancias colectivas referidas a las instituciones intermedias de la economía (Coró y Micelli, 2001). 
El distrito industrial se distingue porque genera redes de empresas especializadas en el ámbito local y modalidades novedosas de aprendizaje técnico que redundan en fuertes niveles de eficiencia colectiva (Schmitz, 1999). En este marco, las firmas no operan de modo aislado, sino que nacen y se desarrollan al interior de sistemas productivos localizados con una fuerte división social del trabajo. Ello permite que la pequeñas empresas adquieran las ventajas de economía de escala sin pagar los costos técnicos y administrativos de los grandes establecimientos, lo que permite un desarrollo socioeconómico en el conjunto territorial. Precisamente, el territorio no sólo aparece como base material de la producción, sino también como infraestructura social, institucional y cognitiva que favorece los procesos de integración y especialización de las firmas.

La importancia que alcanza el fenómeno distrital en la economía italiana de los últimos años replantea el rol y las formas de intervención tradicional en política económica. El distrito industrial, aunque se ha visto favorecido por diversos estímulos gubernamentales otorgados a las pequeñas y medianas empresas, no surge como resultado directo de un diseño específico de política. Su desarrollo es más bien el logro de procesos locales de integración económica y social que involucran a diversos actores y conforman un mix virtuoso de cooperación y competencia productiva. Si se parte del supuesto de que la política industrial es esencial para conservar las ventajas competitivas de un país y garantizar la eficiencia de los mercados, el distrito como objeto de intervención supone reafirmar el patrimonio común de economías externas y bienes públicos territoriales (conocimientos productivos, relaciones entre empresas, servicios reales e institucionalidad) desarrollados a lo largo del tiempo. Esto determina la importancia de generar nuevas formas de governance 1 que permitan revalorar la especificidad productiva de los territorios y favorecer procesos de autoorganización competitiva de los actores locales. Así, la experiencia de los distritos industriales puede ser un primer paso en la conformación de una estrategia de política que contemple la dimensión territorial como un nivel relevante para el desarrollo económico y social.

\footnotetext{
${ }^{1}$ Con el término governance se pretende definir un nuevo estilo de toma de decisiones, diferente a los modelos tradicionales más centralizados y jerárquicos, caracterizado por la interacción y cooperación de los diferentes actores públicos y privados en una política pública particular.
} 
El presente trabajo tiene como propósito central analizar al distrito industrial como objeto de política pública. En este sentido, como forma de enmarcar la discusión, primero se describen las principales características estructurales que asume el distrito industrial italiano y su dinámica en el tiempo. En segundo término, se examina la experiencia del distrito teniendo en cuenta la política industrial en los ámbitos nacional y regional. Enseguida se analiza la arquitectura institucional y los distintos actores y niveles que conforman el proceso de decisiones (governance) de la política industrial en el plano territorial, con especial énfasis en la experiencia desarrollada en la región del Véneto. Finalmente se hacen algunas conclusiones tentativas sobre el distrito industrial y sus implicaciones en las políticas y procesos de governance territorial.

\section{Características estructurales de los distritos y dinámica en el tiempo}

El distrito industrial se puede definir desde una perspectiva neomarshalliana ${ }^{2}$ como "una entidad socioterritorial caracterizada por la copresencia activa, en un área territorial circunscrita, natural e históricamente determinada, de una comunidad y de personas y de una población de empresas" (Becattini, 1991: 23). Esto resalta la importancia del distrito como entidad territorial de producción y de vida, donde se conjugan en forma relativamente indiferenciada aspectos económicos, sociales, históricos y culturales.

Entre sus principales características podemos citar una elevada división del trabajo entre empresas (en general PyMEs) concentradas espacialmente y especializadas de manera sectorial; procesos de innovación de carácter distributivo; relaciones entre empresas competitivas/colaborativas; vínculos hacia adelante y hacia atrás sustentados en relaciones de mercado y extramercado en el intercambio de bienes, información y recursos humanos; y la relevancia que asume el territorio (apoyo institucional, redes de confianza y capital social) como soporte del desarrollo y la creación y

\footnotetext{
2 Alfred Marshall (1879 y 1920) destaca las ventajas económicas que genera la cercanía entre firmas que permiten potenciar la denominada atmósfera industrial y generar economías externas que puede aprovechar el conjunto empresarial que opera en un territorio: oferta local de materias primas y servicios, creación de un mercado de trabajo especializado, acceso a un flujo de conocimientos técnicos, mercado de maquinarias, etcétera.
} 
difusión de conocimiento (Brusco, 1990; Dei Ottati, 1997; Becattini, 1991 y 1998; Putnam et al., 1993; Rabelotti, 1997).

La consolidación del distrito como modelo de especialización en el ámbito territorial se debió a varios factores. En primer término, el desplazamiento de la actividad manufacturera hacia nuevos territorios del nordeste y centro del país (zonas que tradicionalmente presentaban las características de un sistema productivo poco desarrollado) que lograron un fuerte impulso a partir de los años setenta y que se unió a un proceso de especialización y predomino de las firmas de menores dimensiones. Así, de un desarrollo de naturaleza fordista basado en la gran empresa y la industria pesada, centrado en el llamado Triángulo Industrial (regiones de Lombardía, Piamonte y Liguria), se fue dando paso a un modelo productivo con preeminencia de pequeñas y medianas empresas especializadas en bienes tradicionales y localizadas sobre todo en las regiones del Véneto, Emilia Romagna, Toscana y Marche. ${ }^{3}$ Entre los factores que promovían este cambio se pueden mencionar la crisis por el aumento del precio del petróleo y los conflictos laborales de esos años, que trastocaban la estructura de costos de las grandes firmas y las obligaba a subcontratar parte de su producción en pequeños establecimientos, en el marco de una organización productiva más desintegrada que respondía a mercados más diferenciados e inestables. Este agotamiento paulatino del patrón de desarrollo fordista del norte italiano se hizo evidente con la pérdida de más de 150,000 puestos en la industria pesada en el ámbito nacional en el periodo 1971-1981, y el crecimiento del empleo en más de 400,000 puestos en pequeñas firmas (10-49 ocupados) especializadas en productos tradicionales. ${ }^{4}$

En segundo lugar, el incremento de los ingresos de la población italiana y de los principales países europeos permitió un aumento de la demanda interna y externa de bienes de consumo y de lujo (indumentaria, calzado, muebles, electrodomésticos y distintos aparatos mecánicos, joyas y objetos de valor, tejidos).

\footnotetext{
${ }^{3}$ Estas regiones conforman lo que se ha denominado Tercera Italia, en contraposición al noroeste más desarrollado en lo económico y al sur más postergado. En los últimos años, aparecen nuevas dinámicas regionales que muestran sobre todo un fuerte crecimiento en las regiones del nordeste (Véneto, Friuli Venecia Giulia y Trentino Alto Adige). Véase Corò (2003) y Anastasia y Corò (1996).

${ }^{4}$ Las regiones del nordeste y centro de Italia se han desarrollo con base en la difusión de sectores tradicionales como calzado, muebles, pieles, anteojos, orfebrería/ joyas, mármol y cerámica, ligados fuertemente a la industria metalmecánica. Estos sectores aparecen para la época como verdaderos nichos de mercado internacional y con el tiempo logran alcanzar altos estándares de calidad e innovación.
} 
Esto se dio en un escenario de cambio nominal favorable de la lira y de crecimiento de ventas en el exterior a partir de una fuerte política de precios. Por otro lado, el estímulo hacia el desarrollo de empresas de pequeña envergadura se vio favorecido por un contexto de democracia económica basado en una distribución más equitativa de la renta y el apoyo institucional local de administraciones gubernamentales, organizaciones empresariales, viejas cámaras de comercio y bancos cooperativos que permitió la reducción de barreras de ingreso para nuevas firmas y animó un clima de confianza y participación activa en el proceso de crecimiento económico (Saba, 1998; Bertini, 1999).

En sus inicios, estos establecimientos manufactureros -ante la declinación de la ocupación agrícola y proliferación de una industrialización difusa- conformaban producciones de fuerte contenido artesanal y familiar, que gradualmente extendieron su mix de productos para satisfacer las nuevas necesidades de consumo. Al ampliarse las oportunidades de mercado se incrementaron concomitantemente los niveles de inversión empresarial y de actividad industrial. Estos factores se desarrollaron de manera acelerada en los años ochenta y consiguieron un mayor impulso a partir de los procesos de integración económica de la Unión Europea en la década de los noventa. En este contexto, los territorios del nordeste y centro de Italia, en especial el Véneto, exhibieron una tasa de ocupación de la industria manufacturera que creció una tercera parte en el periodo 1971-1996, así como una tasa de desempleo promedio en los niveles más bajos de los países de Europa (3.9\% para 2002) y notablemente inferior al resto estimado de las regiones de Italia (10.7\% para el mismo año).

\subsection{Especialización productiva made in Italy, pequeñas empresas $y$ distritos industriales}

El proceso de industrialización desarrollado en Italia desde la posguerra en adelante determinaría una clara especialización productiva centrada en algunas ramas manufactureras. El complejo de sectores denominados Made in Italy -sistema moda (textil, vestimenta, calzado, piel y cuero, anteojos, joyería), sistema casa (madera, muebles y equipamiento, cerámica, vidrio, electrodomésticos, tuberías), alimentario mediterráneo y mecánica ligera- participa en los últimos años con casi 3.8 millones de empleos, lo que constituye $70 \%$ de la ocupación manufacturera italiana (Quadrio-Curzio y Fortis, 2000). Precisamente los dis- 
tritos industriales se especializan en estos sectores y contribuyen con más de 224,000 firmas y 2'170,000 empleados dentro de los 199 sistemas locales identificados por el ISTAT (Instituto Nacional de Estadísticas de Italia).

Este tipo de especialización liviana alcanza también una fuerte incidencia en el comercio internacional desde mediados de los noventa, con una propensión a la exportación sobre el PIB de 9\% y una participación en el mercado del G7 de 66\% en calzado, más de la mitad en la industria del cuero, la joyería y la cerámica y un tercio en los sectores de vestimenta, muebles y equipamiento, así como mecánica ligera. En este sentido, la evolución de las exportaciones italianas en los últimos años muestra una participación fuerte de estos sectores, aunque con una muy ligera caída en términos porcentuales en el periodo más reciente (cuadro 1).

\section{Cuadro 1}

Exportaciones italianas por sector según cuota de mercado (porcentajes)

\begin{tabular}{lrrrr}
\hline & 1997 & 2001 & 2003 & $2004^{*}$ \\
\hline Textil e indumentaria & 8.2 & 7.7 & 7.6 & 7.4 \\
Calzado y productos en piel & 15.9 & 15.9 & 15.6 & 15.1 \\
Productos en goma y plástico & 7.0 & 6.4 & 6.6 & 6.4 \\
Muebles & 18.3 & 15.1 & 14.1 & 13.3 \\
Electrodomésticos & 16.2 & 14.5 & 14.4 & 13.8 \\
Vidrio, cerámica, material & & & & \\
de construcción & 13.4 & 12.5 & 12.1 & 11.8 \\
Productos metálicos & 4.9 & 4.9 & 5.0 & 5.0 \\
\hline
\end{tabular}

*2004: enero-septiembre.

Fuente: Eurostat e ISTAT.

Es importante hacer hincapié en un dato que tiene que ver con que casi $85 \%$ de los empleados en estos sectores se encuentran ocupados en empresas PyMEs de menos de 200 personas. En estos términos, la combinación entre especialización de bienes tradicionales y predominio de pequeñas y medianas empresas, en la actualidad caracteriza a toda la estructura económica italiana, en relación con otros países desarrollados. Como se indica en el cuadro 2, más de la mitad de los empleos en el sector manufacturero están en firmas con menos de 50 personas, donde destancan de manera comparativa el segmento más de tipo artesanal (uno a nueve empleados), y casi tres cuartas partes de la ocupación en empresas de hasta 250 trabajadores. 


\begin{tabular}{|c|c|c|c|c|}
\hline \multicolumn{5}{|c|}{$\begin{array}{l}\text { Cuadro } 2 \\
\text { Distribución de ocupados por dimensión de la empresa para } \\
\text { países desarrollados } \\
\text { (porcentajes) }\end{array}$} \\
\hline & Italia & Alemania & Francia & Inglaterra \\
\hline 9 ocupados & 23.3 & 7.4 & 8.1 & \\
\hline & 29.2 & 14.3 & & \\
\hline s & 18.9 & 15.8 & 21.2 & 21.7 \\
\hline Más de 250 ocupados & 28.6 & 62.5 & 53 & 55.5 \\
\hline Total & 100 & 100 & 100 & 100 \\
\hline
\end{tabular}

Fuente: Eurostat.

Este menor tamaño de las unidades productivas, fenómeno denominado enanismo industrial, puede verse al mismo tiempo como ventaja y desventaja. Es claro que en un contexto de ciclos productivos sumamente fragmentados, las firmas pequeñas especializadas pueden ser más competitivas y flexibles para responder a las demandas cambiantes del mercado. En las regiones del nordeste y centro del país la dimensión actual promedio de la industria manufacturera no alcanza los 15 ocupados, lo que significa una diferencia importante en comparación con décadas anteriores donde las empresas más grandes (de más de 100 empleados) tenían mayor incidencia sobre el total de firmas. El tamaño tiene, por tanto, un sentido muy diferente si se le ubica en un escenario de articulación productiva y elaboración muy particular, como puede ser el distrito, de otras de formas de organización de la producción más estandarizadas y de carácter masivo.

Es necesario destacar, no obstante, que las economías de escala siguen teniendo una fuerte relevancia en un marco donde aumenta el rol del conocimiento en los procesos productivos y se requiere de fuertes inversiones. Las grandes empresas poseen las dimensiones suficientes como para absorber los costos y riesgos que supone este tipo de iniciativas. En el caso de las pequeñas firmas, la única posibilidad de compensar estas desventajas es operar de manera articulada en sistemas productivos y redes de división social del trabajo. Precisamente el distrito industrial se ha presentado como una de las formas más desarrolladas en este sentido, teniendo como ejes a empresas fuertemente especializadas y al territorio como base material, social, institucional y cognitiva.

De esta manera, las economías externas adquieren gran importancia a partir de un núcleo de competencias técnicas (knowhow, know-what) y sociales (know-who) radicadas en el ámbito 
territorial, la difusión de capacidades y saberes, la generación continua de innovaciones y observaciones imitativas, la posibilidad del contacto cara a cara y confianza en las relaciones de compra-venta, el desarrollo de complementariedades entre industrias especializadas por proceso o producto, la ampliación del trabajo especializado en las distintas fases del proceso productivo, la movilidad de recursos humanos y relaciones de una empresa a otra junto al desarrollo de spin-off y el fomento de la capacidad emprendedora (Corò, 2002; Grandinetti y Tabacco, 2003; Bellandi, 1999).

\subsection{Innovación, circulación del conocimiento y utilización de nuevas tecnologías}

Desde sus inicios, la difusión de la innovación como proceso de aprendizaje cumple un papel relevante al interior de los distritos. La permanente búsqueda y experimentación de nuevos materiales y técnicas para la producción permitieron mejorar las características de los bienes finales mediante un proceso de adaptación y perfeccionamiento continuo en el tiempo. En este marco, el learning by doing (mejoramiento unido a la producción) y el learning by using (mejoramiento ligado al uso del producto) conformaron partes esenciales de este innovador proceso de aprendizaje..$^{5}$

En un contexto de cambio tecnológico permanente como el actual, y ante los altos costos de inversión en conocimiento, la organización del proceso productivo en fases permite la fuerte especialización de las empresas de los distritos en sistemas integrados de relaciones que generan redes de competencia y permiten repartir en gran número de productos los costos hundidos de las inversiones. La presencia de una multiplicidad de redes, que varían según el sector en términos de características técnicas del producto, mercados y destinatarios finales, aparece entonces como uno de los elementos de mayor fortaleza del distrito. Esto supone la importancia de pensar no meramente en la especialización en un producto, sino en el conjunto de la cadena productiva. Así, el distrito puede crear un contexto favorable a la innovación en la medida que constituya una plataforma de gestión del conocimiento y defina un modelo de relaciones generativas

${ }^{5}$ Estos saberes de distinto tipo se van acumulando a lo largo del path dependence de los agentes, sean éstos individuales o colectivos, y se manifiestan en la creación de activos tangibles e intangibles que resultan centrales en el proceso de competencia. 
que permita crear nuevos agentes, nuevas reglas y nuevas instituciones (Tattara, 2003).

Esto plantea la importancia de ver al distrito industrial como un laboratorio cognitivo, es decir, como un sistema cuya función básica es aprender. El foco sobre el conocimiento y los assets intangibles, considerados como bienes públicos locales, son cuestiones centrales. El Made in Italy representa una porción importante del contenido intangible de la producción italiana, en particular en los sectores tradicionales (por ejemplo, capital humano y de relaciones, innovación, calidad, tradiciones, habilidades y conocimientos tácitos). En ese sentido, asumen una enorme relevancia aspectos como: la densidad de flujos de transferencia de conocimiento que alimentan el proceso de knowledge creation y generan un knowledge-based en la formación y reproducción evolutiva del distrito, la importancia que asumen los mecanismos de innovación y correspondiente imitación de producto, el despliegue de spin-off ${ }^{6}$ (a partir del cese de actividades de la empresa original y el nacimiento de nuevas unidades -spin off de primer nivel- o el distanciamiento de estos últimos con la primera -spin off de segundo nivel-, con fuerte contenido replicativoespecializado), la movilidad del recurso humano en un mercado de trabajo extremadamente dinámico permite el paso de personas entre empresas y estimula el knowledge transfer y un proceso de enterprise creation (Grandinetti y Tabacco, 2003; Corò, 2001a).

Al mismo tiempo, en los últimos años las nuevas tecnologías de información y comunicación (TICS) adquieren un papel protagónico como factores de competitividad de las actividades productivas. Desde un punto de vista teórico, se resalta la importancia de las TICs como herramientas para promover una evolución positiva de los distritos. Puesto que el conocimiento y el intercambio de información conforman aspectos determinantes del sistema distrital, es interesante establecer el papel que las TICs pueden jugar en esta nueva etapa. Es claro que el debate sobre la incidencia de este tipo de tecnologías se vincula a discusiones más amplias del distrito como: mantener la tasa de innovación para competir a nivel internacional, la integración en redes internacionales y la demanda de nuevos servicios, en especial logística (Tattara y Volpe, 2003).

\footnotetext{
${ }^{6}$ La literatura plantea que el proceso clave en el pattern formativo lo constituyen los spin-off generados por la empresa originaria, que presenta por tanto el rol de empresa seminal o de matriz de empresarialidad.
} 


\section{Cuadro 3}

Las TICs en los distritos italianos. Años 2000 y 2003 (porcentajes)

\begin{tabular}{lcc}
\hline Tecnología & 2000 & 2003 \\
\hline Correo electrónico & 93.8 & 99.3 \\
Sito Web & 72.8 & 91.1 \\
Corporate banking & 68.6 & 76.3 \\
Broadband & 14.9 & 74.1 \\
ISDN & 85.9 & 70.8 \\
ERP & 21.2 & 34.8 \\
Groupware & 10.9 & 22.5 \\
EDI & 11.1 & 15.7 \\
Videoconferencia & 7.9 & 13.4 \\
Comercio electrónico & 1.1 & 10.9 \\
\hline
\end{tabular}

Fuente: Micelli et al., 2006a.

Si examinamos la intensidad con que las nuevas tecnologías participan en el funcionamiento del distrito, es posible advertir en forma resumida algunas evidencias empíricas. Un análisis comparativo de 557 firmas de los 33 distritos industriales más importantes de Italia para los años 2000 y 2003 nos muestra un incremento general del uso de estas tecnologías (cuadro 3). Esto se produce en las firmas más dinámicas, por lo general de tamaño medio y como parte de estímulos externos. En efecto, en un marco de internacionalización creciente, las empresas tienden a replicar hacia afuera el mismo modelo de comunicación y de relaciones del sistema local. Esto llevó en el 2000 a dar un amplio espacio a los recursos e instrumentos de comunicación más simples (encuentros, teléfono, correo electrónico, fax,) por sobre los recursos e instrumentos más sofisticados (ERP -enterprise resource planning-, groupware, videoconferencia, comercio electrónico). Sin embargo, esta brecha que existe claramente entre las tecnologías se ha venido reduciendo en el 2003, mostrando importantes incrementos en la utilización de ERP y groupware (Micelli et al., 2003 y 2006a).

En consecuencia, es claro que el papel de las Tics en los distritos se debe definir aún con mayor precisión y su desarrollo estará vinculado a los diversos desafíos estructurales y de organización que los sistemas distritales deberán asumir en el nuevo contexto competitivo. Las perspectivas de los últimos años muestran una tendencia general de crecimiento de todas las soluciones tecnológicas en los distritos, con altas tasas de adopción de ERP y groupware por parte de las empresas más dinámicas, aun- 
que también se evidencia que las otras firmas perciben la necesidad y las ventajas de usar este tipo de dispositivos.

\subsection{Internacionalización, redes y deslocalización de los distritos}

El fenómeno de la internacionalización tiene fuerte incidencia sobre el funcionamiento actual de los distritos industriales. El aumento de los intercambios mundiales y financieros, la difusión de nuevas formas organizativas de producción, el fin de la división del mundo en dos bloques antagónicos y el surgimiento de nuevos países que se integran fuertemente a la economía mundial (Europa Central y del Este, India y China), establece un escenario caracterizado por mercados más amplios y la necesidad de nuevas estrategias y cambios estructurales de las empresas distritales en términos de diferenciación, innovación y redes a escala global.

Los procesos de integración internacional de pequeñas y medianas empresas constituyen un fenómeno reciente y no del todo desarrollado en el análisis económico. En los distritos los procesos de internacionalización se desarrollan de forma distinta a la firma multinacional. Si en este último caso el proceso de internacionalización es de tipo jerárquico, para el distrito se manifiesta a través de la construcción de redes cooperativas de intercambio y abastecimiento. Una estrategia de integración productiva a escala internacional puede desplegarse a través de acuerdos comerciales e industriales, licencias a corto término, proyectos de cooperación tecnológica y redes estratégicas. La integración también se puede dar a partir de la decisión de confiar una fase de la elaboración a empresas del exterior y adquirir un carácter más operativo. Como se puede apreciar, en esta situación se genera una integración internacional de la producción a través de relaciones de mercado que no necesariamente comportan un manejo de la cadena de proveedores. Esta tendencia se puede reforzar con procesos como el outsourcing y el networking, la difusión de tecnologías de red y el empleo de sistemas de e-business (Corò, 2001a; Tattara y Volpe, 2003).

Frente a un marco de competencia intensificada las estrategias de las firmas distritales, no excluyentes, principalmente son tres: 1) el desplazamiento de la producción hacia los segmentos más altos del mercado -alta calidad, lujo-, con mayor contenido de conocimiento, 2) la deslocalización de fases de producción en países que presentan bajo costo de mano de obra y 3) el acceso a más mercados externos. Estas dos últimas estrategias se vinculan de manera directa con el proceso de internacionalización. 
Generalmente, las firmas de los distritos industriales han ampliado su acceso a los mercados desarrollando alianzas con un leader mundial para acceder a sus redes de comercialización y distribución, o afirmándose a través de una empresa de medianas proporciones para que sirva de interfaz con el mercado. Los procesos de deslocalización, por su parte, se pueden dar de distintas maneras. Es posible deslocalizar a través de la cesión de instalaciones en la base doméstica y la relocalización en terceros países. También es posible relocalizar o utilizar una instalación en el exterior para expandir la producción que en el territorio de origen no es posible o conveniente estimular. De la misma forma, un proceso de deslocalización se puede dar mediante la decisión de confiar una fase de la elaboración a empresas externas o adquirir una línea de productos o componentes, primero realizados al interior de la empresa, por proveedores ubicados en localidades remotas. Justamente una sustitución de proveedores locales con proveedores externos entra en la categoría de deslocalización en cuanto se plantee un desplazamiento de la actividad laboral hacia otro país (Corò, 2001a).

El principal objetivo de las empresas para descentralizar la producción en países en desarrollo ha residido inicialmente en aprovechar las oportunidades que se presentan en cuando a costos del trabajo. ${ }^{7}$ Esta estrategia en la cadena de valor del Made in Italy sólo se desarrolla en algunas fases específicas -labour intensive-, mientras otras (donde se necesita mayor contenido de calidad manufacturera) requieren un crecimiento de las inversiones de la base doméstica. Por otra parte, en muchos casos la deslocalización se ha dado por la falta de mano de obra local, y en ese sentido se presenta como una oportunidad y una necesidad. De esta forma, es importante ver a este proceso no sólo como un transferencia de actividad de un territorio a otro, sino más bien como una transformación más integral de la organización de procesos globales de producción (Corò y Volpe, 2003).

Para el periodo 1986-2005, el retraso en la expansión internacional de las empresas italianas de mayor tamaño se compensó con la inserción en los mercados de las PyMEs. En estos térmi-

\footnotetext{
${ }^{7}$ Ejemplos de este proceso son el distrito del calzado de Verona (deslocalización de fases de la producción en Bosnia, Rumania, India y tiene previsto también en China) y el de ropa deportiva de Montebelluna (especialmente Rumania, provincia de Timisoara). Debido a distintos problemas generados en el terreno práctico, actualmente se plantean perspectivas más amplias que involucran la importancia de lo institucional, lo logístico y lo cultural en el desarrollo de nuevas formas de integración productiva a escala internacional.
} 


\section{Cuadro 4}

Evolución de la participación italiana en el exterior, 1986-2005 (porcentajes)

\begin{tabular}{lrrr}
\hline & 1986 & 1996 & 2005 \\
\hline UE 15 & 43.2 & 39.9 & 31.9 \\
CEEC* & 0.6 & 20.8 & 27.0 \\
Otros europeos & 5.2 & 3.2 & 3.3 \\
África & 10.2 & 10.9 & 9.8 \\
Norteamérica & 14.5 & 8.2 & 7.2 \\
América Latina & 19.4 & 9.4 & 8.9 \\
Asia & 6.7 & 8.2 & 11.1 \\
Oceanía & 1.1 & 0.7 & 0.8 \\
Total & 100 & 100 & 100 \\
\hline
\end{tabular}

"CEEC: países de Europa Central y del Este.

Fuente: Labory, 2006.

nos, se evidencia una fuerte tendencia a la deslocalización en los países de Europa centro-oriental (que comienza con la caída del Muro de Berlín), junto con una fuerte disminución desde 1986 de la participación en América Latina y un aumento de las inversiones en Asia y China particularmente (cuadro 4).

Un análisis de la internacionalización reciente de 764 firmas de 45 distritos industriales importantes de Italia, muestra un nivel de exportación promedio de 44\% sobre la producción y una creciente capacidad para controlar mercados finales o participar mediante asociaciones comerciales, redes de franquicias y puntos directos de ventas. La geografía de la exportación destaca una fuerte orientación hacia las economías emergentes. Las exportaciones alcanzan importantes niveles en la Unión Europea (Alemania y Francia a la cabeza) y Estados Unidos, pero también en Rusia y China, crecientes mercados también de bienes de lujo. En los mercados en alza, la internacionalización de la producción involucra a $28.7 \%$ de las empresas. Ese valor es aún más alto si se examina el nordeste y los distritos industriales vinculados a los sectores de maquinaria y moda (Micelli et al., 2006b).

La dinámica de internacionalización en las regiones con fuerte contenido de distritos, como por ejemplo las del nordeste, presenta niveles acentuados respecto de la media italiana, donde desde principios de la década de los noventa el crecimiento de las exportaciones fue sumamente sostenido. Este desarrollo se acompañó de una elevada dinámica de las importaciones, en un marco de redireccionamiento de los intercambios comerciales caracterizados por la pérdida de peso relativo de los países de la 
UE y por la sustitución de esta cuota de mercado por los países del Este de Europa. ${ }^{8}$

Estos resultados destacan la importancia que asume la internacionalización de la producción en las estrategias de las firmas. A diferencia de lo que ocurre con las grandes empresas, los distritos de pyMEs no sólo invierten directamente en el exterior (inversiones extranjeras directas, IED), también están interesados en impulsar redes locales de distribución en países extranjeros. De la misma forma, aunque todavía es un proceso lento e incipiente, la tendencia hacia la internacionalización no supone la declinación del papel que tienen los sistemas locales italianos en la competencia de las firmas y territorios.

En este sentido, en los últimos años la internacionalización de los distritos adquiere un papel relevante y plantea distintas perspectivas para los territorios. La posibilidad de renovar la base económica del distrito y exportar un modelo de desarrollo aparecen como los aspectos más positivos del proceso. No obstante, se aprecian dificultades para las PyMEs que no están en contacto directo con los mercados y tienen bajas competencias tecnológicas. De este modo, la firma distrital deberá renovar sus formas tradicionales de inserción internacional, con base en actividades manufactureras, para dar paso a modelos de innovación en la gestión, la generación de nuevas competencias internas y la creación de valor en el ámbito territorial.

\section{Políticas para los distritos industriales y nuevas instituciones de governance}

A partir de lo anterior es posible afirmar que el distrito industrial alcanza un papel relevante en el desarrollo económico italiano de las últimas décadas. Su despliegue, beneficiado de manera indirecta por distintas legislaciones e instrumentos a favor

\footnotetext{
${ }^{8}$ Si se toma el caso del sistema productivo local textil-indumentaria de la provincia de Vicenza, en la región del Véneto, el conjunto moda alcanzaba para 2001 una tasa de exportaciones de alrededor de $30 \%$ sobre el total provincial, contra valores que oscilaban en $25 \%$ para 1993 (esto constituye un valor muy importante si se considera que la provincia, en el mismo periodo y depurando el dato del crecimiento interno de los precios, obtiene $66 \%$ en sus exportaciones). Al mismo tiempo, en ese lapso creció la tasa de importación del sistema moda a más del doble, pasando de $12 \%$ a 25\%. Por otro lado, los países europeos disminuyeron sus cuotas respecto de las exportaciones vicentinas y se sustituyeron por cuotas de exportación a los mercados de Europa Centro-Oriental, en especial Rumania, pasando de $4 \%$ a $10 \%$. Esto se manifestó, a su vez, en intercambios comerciales con fuerte incidencia de las importaciones, que indicarían un proceso de integración no sólo comercial sino también productivo (Corò y Volpe, 2003).
} 
de la empresa individual y familiar, ${ }^{9}$ muestra en términos de interacción y especialización productiva un carácter predominantemente endógeno. Al mismo tiempo, lo indeterminado de sus límites y su continua transformación en el tiempo ha generado dificultades para circunscribirlo en términos de política pública. Por un lado, el distrito conforma un entramado de relaciones entre empresas y de vínculos en el mercado de trabajo que no tiene límites precisos sobre el territorio (como los administrativos) y que se van modificando permanentemente. Por otro, aparece como un conjunto de competencias y de especializaciones productivas en continua evolución (con base en la innovación incremental y la adaptación flexible), en el marco de una dinámica de cooperación y competitividad junto a procesos de aprendizaje técnico e institucional.

En los últimos tiempos la importancia del distrito y del desarrollo económico localizado plantean un nuevo marco para la política industrial y sus áreas de intervención, en un contexto de federalismo y creciente significación de los gobiernos regionales italianos. En consecuencia, el análisis del distrito como objeto de política sugiere considerar las distintas etapas que ha atravesado la política industrial italiana hasta la actualidad, el tipo de marco institucional y los actores predominantes, las diversas acciones en materia de política regional y los distintos niveles que conforman el proceso de governance en el plano territorial.

\subsection{Supuestos, lineamientos y etapas de la política industrial italiana}

La política industrial italiana ha pasado por distintas etapas a lo largo de estas últimas décadas y, recientemente, se ha enfocado en la cuestión distrital. Una primera etapa se desarrolla desde fines de la segunda Guerra Mundial hasta fines de los setenta y se caracteriza porque es una política industrial de neto corte sectorial. La segunda se manifiesta desde los años ochenta hasta principios de los noventa y sus principales rasgos son la orientación hacia los factores horizontales de la producción. Finalmente, una tercera etapa que abarca el resto del decenio hasta la

\footnotetext{
${ }^{9}$ Nos referimos a las facilidades para el artesanado en 1952, la ley Sabatini de 1965 -que introduce importantes incentivos para las inversiones en máquinas, herramientas y otros instrumentos públicos a favor de la empresa artesanal (fondi artigiancassa) - y el desarrollo de consorcios promocionales (participación en ferias, misiones comerciales, etc.) en 1989 y consorcios de crédito a través de varios programas de la ley 371/1991.
} 
actualidad se distingue por el impulso al ambiente territorial (económico e institucional) donde se desenvuelven las firmas.

La política industrial que se establece desde los años cincuenta hasta fines de los setenta, como el resto de los países europeos, partía del supuesto de que cada gobierno nacional debía guiar su propia industria. El objetivo central estaba dirigido a constituir una estructura industrial del país que fuera particularmente fuerte en los sectores considerados estratégicos (campeones nacionales). Esta política planteaba la reconversión de los sectores maduros (textil y vestimenta, siderurgia, papel) y el lanzamiento de nuevos sectores con una demanda en crecimiento rápido y considerados clave para el desarrollo económico (electrónica, química, automatización industrial, infraestructura de transporte, telecomunicaciones). El centro de esta política de carácter selectivo era la empresa de grandes dimensiones, con producciones de masa y estandarizadas y fuerte concentración en el Triángulo Industrial (Turín, Milán y Génova), donde la presencia de industria ya era importante antes de la primera Guerra Mundial.

El Estado establecía así objetivos a través del estudio de sectores, sobre la base de una participación importante de las élites tecnocráticas nacionales y el predominio de una visión de la innovación como proceso de transferencia lineal con la participación predominante de laboratorios, instituciones importantes de investigación, universidades y grandes empresas. La crisis del petróleo y los escasos resultados obtenidos con este tipo de política (no se logró contener el déficit de la balanza comercial, no se generó un verdadero proceso de sustitución de importaciones en estas áreas y se perdieron recursos al apoyar a grandes empresas, como Olivetti, con problemas para mantenerse en el mercado) llevaron al desarrollo de nuevas políticas a partir de los años siguientes (Gurisatti, 2003).

La crisis del sistema industrial occidental de fines de los setenta y el predomino paulatino de programas de corte liberal, determinarían para la década de los ochenta un nuevo tipo de política orientada no a sectores específicos, sino al conjunto del sistema productivo. Con la importancia gradual que adquiría el Made in Italy y en un marco de ajuste estructural de la economía -privatizaciones y reestructuración de las empresas públicas- se potenció la orientación hacia los factores horizontales de la producción (capacitación, investigación, infraestructura, financiamiento PyмE). La intervención se centró en el contexto externo de la empresa y se potenció el desarrollo de agencias de servicios 
en temas como formación, innovación, estructuras locales de asistencia, exportaciones, etcétera.

A partir de 1992, con la última devaluación competitiva de la lira y la ratificación del Acuerdo de Maastricht, se dio un proceso de transferencia de poder de dirección a las regiones y un marco de gestión concertada respecto de las inversiones públicas (con fuerte participación de las organizaciones empresariales y de la sociedad civil). Esto se complementaba con el predominio del nivel comunitario supranacional en los grandes ejes de política establecidos y el desarrollo de un nuevo marco legislativo de intervención. Así, los primeros años de la década de los noventa (en particular el periodo 1990-1993) plantearon un cambio sustancial en el desarrollo de políticas industriales. Los trazos centrales de política industrial se decidirían de ahora en adelante en el marco de la actual Unión Europea y mediante distintas legislaciones se daría fin a la intervención extraordinaria en las áreas deprimidas (sur del país) y se estimularía el desarrollo de las pequeñas y medianas empresas en conexión con políticas hacia la innovación (Lanzetti y Marchonatti, 2002; Gurisatti, 2003).

En el año 1993, la ley 317/1991 para el desarrollo de las pequeñas empresas aparecería como la primera intervención de carácter orgánico a favor de la firma de menor tamaño en articulación con su entorno territorial. En la formulación de su artículo 36, "Interventi per l'innovazione e lo sviluppo delle piccole imprese", esta ley plantearía como hecho relevante el sostenimiento del distrito industrial, constituyéndose en una de las iniciativas de política industrial enteramente dirigidas bajo la responsabilidad de las regiones. ${ }^{10}$ Con la entrada de Italia al euro, el mayor protagonismo de las regiones y las restricciones en las cuentas públicas nacionales la política industrial italiana se dirigiría entonces al estímulo de economías externas y servicios a través de prácticas concertadas en el ámbito territorial en los diferentes sectores productivos.

De este modo, las regiones abandonarían progresivamente la asistencia directa sobre la economía a favor de acuerdos negocia-

\footnotetext{
${ }^{10}$ La ley 317/191, en su artículo 36, transfiere a las regiones los instrumentos para el desarrollo de los distritos. En este contexto, las regiones pueden: 1) reconocer jurídicamente como distritos a los sistemas locales del trabajo que presenten índices de especialización sectorial y de densidad de empresas sensiblemente superior a la media nacional y 2) desarrollar diversas acciones de intervención sobre los distritos y sistemas productivos locales. Sin embargo, esta legislación recibiría sucesivas modificaciones en los años subsiguientes, producto de las dificultades para definir en términos operativos la noción de distrito.
} 
dos localmente con las partes. Más allá de los resultados positivos que muchas de estas experiencias han tenido, es necesario aclarar que la lógica predominante de funcionamiento técnico y de las inversiones públicas siguió teniendo un carácter más de tipo topdown (de arriba hacia abajo). Precisamente, la dinámica de los distritos industriales responde en general a una lógica de autoorganización y gestión de los recursos que opera desde la base, bottom-up, y se va extendiendo a niveles superiores. Al mismo tiempo, las disposiciones legislativas emanadas de las regiones y las distintas políticas que se desarrollaron hacia fines de los noventa tuvieron efectos muy limitados para estimular la competitividad de los distritos frente al clima de internacionalización creciente. Esto resalta la necesidad de nuevos instrumentos de política que valoren los activos intangibles de los distritos y contemplen la evolución del modelo competitivo de las empresas.

\subsection{Nuevos postulados de politica orientados al desarrollo de los distritos industriales}

La crisis del modelo tradicional de intervención, en términos de centralización tecnocrática y transferencia top-down, plantea la necesidad de un nuevo tipo de política industrial que valore las particularidades de los distritos y sus territorios en un cuadro de competitividad más amplio. Esto supone, por un lado, la importancia de desplegar acciones para la innovación que den cuenta del contexto territorial, más que de las firmas individualmente. De la misma forma, se requieren nuevos modelos de governance que contemplen el rol activo de los actores e instituciones locales en la generación de proyectos superadores frente a un contexto acelerado de cambio. Una política industrial para los distritos en el plano regional debe centrarse, por tanto, en el desarrollo de acciones de naturaleza estratégica para impulsar las iniciativas de los actores locales sobre temas como innovación, internacionalización y articulación productiva. Así, los ejes de sostenimiento regional para iniciativas de corte local podrían abarcar desde proyectos comunes de investigación y transferencia tecnológica, el armado de redes productivas fuertemente especializadas, incentivos al desarrollo de patentes y el armado de marcas de calidad, como la difusión de tecnologías de red, la creación de nuevas empresas, servicios para la internacionalización y logística, formas novedosas de financiamiento y acciones en materia de marketing territorial (Corò, 2001b). 
En estos términos, el distrito como objeto de política se debe contemplar como un conjunto de actividades que generan valor en relación con la producción de bienes y servicios particulares. En un marco donde se reinterpretan los presupuestos tradicionales de intervención, las políticas para el distrito requieren que se desarrollen y fortalezcan las economías externas y los bienes públicos territoriales. Esto resalta la importancia de garantizar servicios reales a disposición de la mayor cantidad de empresas del territorio, generar redes de innovación (desarrollando lenguajes comunes de producción y estandarización), brindar infraestructura material e inmaterial y promover proyectos de cooperación institucional (Nonaka y Takeuchi, 1995). De esta forma, las intervenciones serán exitosas en la medida que puedan impulsar procesos creativos y la generación de nuevos conocimientos a través de un juego de animación de sujetos de naturaleza diversa (privados, redes de empresas e instituciones, formas mixtas) y el desarrollo de mecanismos de autoorganización competitiva (Schmitz, 1999; Lane, 2002; Florida, 2002; Becattini, 2003).

La arquitectura institucional para el desarrollo de una política industrial regional con eje en los distritos y los sistemas productivos locales se presenta entonces como un elemento de fundamental importancia. La ley 317/191 permitió distinguir distintos niveles de actuación. Un primer nivel lo representa la región, que cumple un papel de dirección general y articulación en cuanto al financiamiento propio, nacional y comunitario, a la vez que participa en la aprobación de lineamientos y proyectos de desarrollo local, monitoreo y control de resultados. Después aparece el denominado comité del distrito (con distintos nombres según la región) que se presenta como una estructura autónoma creada por los actores económicos locales (asociaciones empresariales, entidades gubernamentales, cámaras de comercio, centros de servicios, escuelas técnicas, universidades, bancos locales, etc.) cuyas funciones tienen que ver con la representación y proyección del distrito. Finalmente se destacan los objetivos estratégicos en materia de política industrial definidos por el comité del distrito como Planos de desarrollo local. En esta instancia se definen las metas en los distintos ejes (innovación y calidad, empleo, formación, estímulo del territorio -marketing territorial-, medio ambiente) como los instrumentos para alcanzarlas. Este proceso implica considerar los temas prioritarios que deben ser financiados, los actores y los proyectos de interés común que se van a sostener con contribuciones de la región (Corò, 2001c y 2002). 
La aplicación de este tipo de funcionamiento institucional ha tenido distintos resultados según las regiones. Es claro que la compatibilidad entre las esferas regional y local se presenta como un aspecto central cuando se considera un marco institucional que haga hincapié en los distritos y las aglomeraciones productivas territoriales. Si bien la responsabilidad de individualizar los temas de política industrial deben quedar en manos de las fuerzas locales, esto tiene que darse en el contexto de una estrategia regional de desarrollo articulada en los ámbitos nacional e internacional. La complementariedad entre las iniciativas locales y los principales lineamientos esbozados por la región determinan una mejor asignación de los recursos, así como una coherencia territorial efectiva que evite duplicaciones o favorezca acciones aisladas.

\subsection{La experiencia de la región del Véneto}

La región del Véneto constituye un ejemplo muy interesante en la definición de aspectos institucionales y de governance que caracterizan a un nuevo tipo de política industrial con énfasis en la temática del distrito. La discusión sobre las principales características que debe asumir la intervención en el escenario actual tiene raíces particulares vinculadas con las siguientes cuestiones: 1) el hecho de que los recursos disponibles para el modelo de economía difusa (territorio, mano de obra local y posibilidades de innovación) se encuentran en una fase de agotamiento y 2) el fuerte impulso federalista y el poder de las asociaciones empresariales, más protagónicas en el Véneto que en otras regiones italianas, que determinaron demandas muy fuertes de cambio en relación con el sostenimiento de las pymes y la innovación (Gurisatti, 2003).

Actualmente el Véneto se caracteriza porque es una economía regional con plena ocupación (la tasa de desempleo promedio de los últimos años osciló entre 3\% y 4\%), con gran cantidad de empresas (alrededor de 450 mil en los diferentes sectores) y un fuerte modelo de especialización industrial basado en sistemas locales de pequeñas empresas. Justamente, es posible identificar alrededor de 15 distritos que contienen entre 100-150 mil empleados que trabajan en cerca de 10,000 empresas en sectores de especialización distrital (textil, muebles y equipamiento, calzado, piel, anteojos y maquinaria asociada). ${ }^{11}$

${ }^{11}$ Para un análisis del desarrollo económico véneto desde los años sesenta hasta la actualidad, véase Anatasia y Tattara (2003). 
En este contexto, la reciente ley para el reconocimiento de distritos aprobada por la región del Véneto en abril de 2003 concentra su interés en las redes de empresas que intervienen en el territorio. Los ejes centrales de la política se orientan a cofinanciar actividades surgidas desde el propio sector productivo, para generar procesos interactivos y autoorganizados entre los agentes en el marco de un compromiso entre las partes y el desarrollo de metas de cumplimiento efectivo.

La nueva ley regional define al distrito como una entidad territorial caracterizada por una elevada concentración de empresas integradas entre sí en un sistema productivo, donde operan un conjunto de actores institucionales en el sostenimiento de la economía local. Los criterios que determinan la relevancia del sistema productivo tienen que ver con el número de empresas y de ocupados en el territorio, la importancia del entramado en las economías regional y nacional, la radicación histórica de competencias distintivas, la capacidad de innovación y la función estratégica del sistema para el futuro de la región. Por otra parte, como sujetos institucionales con capacidad de actuación aparecen fundamentalmente las administraciones locales, las asociaciones empresariales, los centros de servicios, así como las instituciones educativas y de formación profesional. De esta forma, el concepto de distrito se considera desde una perspectiva amplia donde se incluyen tanto los factores económicos como los aspectos socioinstitucionales que caracterizan a una sistema productivo territorial abierto.

En este escenario se plantea como requisito indispensable que los actores privados e institucionales que forman un distrito productivo elaboren una proyección estratégica y presenten a la región un pacto para el desarrollo distrital. Este pacto, que parte de la idea de distrito como agregación de actores, debe comprender al menos 80 empresas y 250 empleados en el marco de un sistema de integración especializado (o cadena productiva) con la participación de las instituciones locales. Los pasos que se consideran para la constitución del pacto como proyecto incluyen un estado de situación del distrito, los puntos fuertes y débiles, la estrategia de desarrollo planteada a mediano término, los recursos financieros disponibles y las prioridades proyectadas.

La ley contempla que la Cámara de Comercio, por su disponibilidad de datos e información general, valide y acredite los contenidos del pacto. Al mismo tiempo, se prevé que cada sistema productivo local elija un representante de distrito que asumi- 
rá el rol de garante del pacto en la denominada Consulta dei Distretti (que conforma un órgano consultivo del asesor encargado y la junta regional en la gestión de la ley). Este órgano se compone de diversos representantes de los distritos acreditados y se orienta a la preparación de los anuncios y el monitoreo general de los pactos en el ciclo de vigencia de la ley (tres años).

La región, por tanto, reconoce a los distritos no de manera ex ante sino en función de una red de agentes actuantes y capaces de generar una estrategia evolutiva. Una vez definidos éstos, se pasa a una segunda fase donde se formula una convocatoria abierta para la asignación de recursos a proyectos tendentes a satisfacer los objetivos del distrito (la participación es libre para agentes locales y externos). El sostenimiento regional para este tipo de iniciativas supone que los aportes se realizan en la medida que hagan lo propio los sujetos interesados (la contribución pública es de hasta $40 \%$ de los gastos admitidos, contra $60 \%$ que deben solventar quienes participan en el desarrollo del distrito). Así, los criterios generales privilegiados para la aprobación de los proyectos son los siguientes: 1) coherencia respecto de las prioridades estratégicas de la política industrial regional y del pacto de desarrollo local, 2) que los promotores de las iniciativas asuman responsabilidades y contribuyan al financiamiento, 3) la creación de externalidades positivas, 4) la valoración de los recursos y estructuras locales, 5) la participación de la mayor parte de los actores involucrados en la realización del proyecto y 6) el despliegue de sinergias e integraciones con proyectos desarrollados en otros distritos.

Es importante destacar que una vez que se aprueba el pacto para el desarrollo del distrito, esto no supone que los proyectos sean refrendados de manera directa o se asignen recursos a los actores involucrados. Justamente en la etapa de selección y financiamiento de los proyectos (tercera fase del proceso), se deja de lado la posibilidad de que éstos los realicen quienes hacen el pacto distrital. Con esto se intenta involucrar a otros actores vinculados con la esfera productiva y con experiencia técnica. De esta forma se establece un núcleo de validación de los proyectos y se verifica si responden a los objetivos de la política industrial regional y a las indicaciones de los pactos de desarrollo de cada distrito industrial.

Con esta serie de procedimientos se busca asegurar un correcto empleo de los recursos públicos mediante la elección y financiamiento adecuados de los proyectos, la transparencia de la ges- 
tión, la apertura a distintos agentes en la oferta de servicios para los distritos y la participación de la parte privada en las iniciativas. Por otro lado, mediante esta ley el distrito considera no sólo con base en algunas variables estructurales (cantidad de empresas, ocupación, grado de especialización, etc.), sino también en relación con la capacidad de los actores para construir estrategias cooperativas y desarrollar proyectos comunes. La posibilidad de valorar y promover acciones de cooperación entre las empresas y las instituciones locales, intenta superar los límites mostrados en la aplicación de la ley 317/1991 en relación con la creación de una estructura formal de gobierno económico local (el comité del distrito), que con frecuencia contribuye a burocratizar las decisiones y a generar una idea de autoridad técnica superior de gestión sin participación de los actores locales.

Si bien la ley presenta estos aspectos positivos, también es importante considerar algunas interrogantes que surgen en su desarrollo. Por un lado, aparecen dudas respecto al rol final que jugarán efectivamente las asociaciones intermedias -organizaciones empresariales, cámaras de comercio, etc.- en la nueva institucionalidad que la legislación plantea, tomando en cuenta el papel protagónico que han tenido en el crecimiento del distrito tradicional. Otro elemento tiene que ver con el fuerte protagonismo que se le asigna al asesor y a la junta regional que pueden ser determinantes en la suerte que corra el proceso. Por otra parte, la gran amplitud y diversidad de propuestas que se encuadran en el marco del distrito pueden llegar a incluir casos con debilidades en su constitución (que se supone se irán corrigiendo en la medida que la ley entre en una fase plena de aplicación). Más allá de estos puntos, es claro que la legislación constituye un caso innovador en términos de intervención en los ámbitos industrial y territorial. Hasta el momento se han presentado 28 pactos para el desarrollo de distritos, que abarcan desde los tradicionales como Montebelluna (calzado deportivo), Murano (vidrio) y Valpolicella (mármol) hasta nuevos y singulares especializados en packaging, biotecnología y logística), y 115 proyectos (en investigación aplicada, transferencia tecnológica, creación de observatorios y bancos de datos del distrito, participación en ferias internacionales, realización de productos informáticos y promoción de las marcas distritales) con un financiamiento regional de 15 millones para cada ejercicio en el trienio 2003-2005.

Esta nueva legislación permite distinguir distintos planos de intervención en términos de governance. Un primer plano es de 
carácter regional y se vincula fundamentalmente con la evaluación y administración, la definición de recursos para la política industrial y determinaciones en cuanto a los criterios para el financiamiento de los proyectos. Después aparece el nivel local en relación con el desarrollo de los pactos y con la participación en la Consulta dei Distretti. En último término se destacan los proyectos para los distritos, promovidos por actores individuales o colectivos, públicos y/o privados, locales y/o externos.

Estos tres planos se deben entender como componentes de una cadena articulada y flexible, donde la búsqueda de eficacia se centre en el alcance de los objetivos propuestos y no sólo en el cumplimiento formal de los procedimientos. La idea central es generar mecanismos versátiles y de carácter estratégico, con seguimiento técnico en el ámbito regional y de otras instituciones que permitan que los actores locales desarrollen proyectos innovadores de base territorial en el marco del distrito, contemplando su inserción externa. Por tanto, el acercamiento al nivel de governance tiene un carácter estrechamente evolutivo, en cuanto que se basa en una estrecha relación entre la fase de negociación e implementación de políticas y supone procesos dinámicos de aprendizaje, mejoramiento continuo y desarrollo interactivo de los componentes de la red a nivel local, regional e internacional.

\section{Reflexiones finales}

A lo largo de las últimas décadas, el distrito industrial italiano ha atravesado por distintas etapas que lo han consolidado como una de las formas más virtuosas de organización de la producción y desarrollo de modalidades de aprendizaje técnico en el ámbito territorial. En la actualidad los procesos de internacionalización redefinen la lógica tradicional del distrito, alargan la dimensión del territorio (y de sus actores) y obligan a repensar el fenómeno local contemplando la escala global.

En este contexto, organizar una política para el distrito industrial no es tarea sencilla. El distrito existe en la práctica porque los actores económicos locales se identifican en un sistema de relaciones sociales y productivas, donde se combinan distintos valores y un sustrato común de conocimiento técnico. Esto plantea la necesidad de un modelo de política industrial que no sólo tenga una base legislativa adecuada, sino fundamentalmente un sistema concreto de acciones e incentivos a favor de las estra- 
tegias de los actores en el marco de las redes (locales y extra locales) donde se desenvuelven.

Pensar al distrito como sujeto de política supone considerar el legado común de economías externas y bienes públicos territoriales desarrollados en el transcurso de los años. De la misma forma, implica favorecer procesos de autoorganización competitiva de los actores locales que permita dinámicas de adaptación espontánea. Por último, también plantea la importancia de generar nuevas formas de governance que permitan revalorar la especificidad productiva de los territorios y la construcción social de nuevos conocimientos.

En este escenario se hace indispensable construir una visión del distrito que contemple, en un contexto de internacionalización e innovación permanente, la fuente de especialización del distrito (como valor diferencial y de calidad) a partir de los distintos aspectos económicos y sociales que permitieron su crecimiento. Supone también la generación de capital creativo, competencias distintivas, inteligencia institucional y la priorización de un modelo de desarrollo socialmente sostenible y sustentable. En un contexto de competitividad como el actual, una estrategia de este tipo no se puede dejar solamente en manos de unas pocas empresas e involucra un campo de acción para una política amplia del distrito que promueva la generación de conocimiento, fortalezca las dinámicas empresariales y el desarrollo de redes, involucre una serie de servicios e infraestructuras estratégicas y permita la internacionalización del sistema productivo.

En la actualidad estos principios renovados de política en relación con los distritos se encuentran en pleno desarrollo, tal como hemos visto con la región del Véneto. Sus resultados todavía son incipientes, aunque es posible advertir avances en lo que se refiere a la investigación aplicada, la transferencia tecnológica, la institucionalidad establecida y los niveles de participación en el plano internacional. Precisamente la apertura de los distritos genera que, por lo general, el territorio local sea el espacio para el desarrollo de actividades innovadoras de alto valor agregado; el resto de Europa occidental, el lugar para la adquisición de competencias y recursos especializados; los países del Este europeo, el sitio para el despliegue de redes controladas basadas en mano de obra deslocalizada; y Asia y Oriente -particularmente India y China- lugares privilegiados para tomar oportunidades en términos de costos y mercados. 
Este proceso de internacionalización, sin embargo, fortalece empresas más estructuradas de medianas dimensiones y plantea dificultades para las pequeñas firmas de los distritos, especialmente para aquéllas que no están en contacto fluido con el mercado y tienen bajas competencias tecnológicas. De este modo, una respuesta de política industrial estratégica deberá considerar aspectos defensivos y de reposicionamiento y consolidación. En el primero de los casos, supone gestionar en poco tiempo el impacto, incentivando procesos de reestructuración y reconversión industrial para responder a las demandas de mediano y largo plazos. En el segundo, implica promover inversiones en lo que se refiere a formación e investigación en sectores de alto contenido en conocimiento (informática, biotecnologías, genética, robótica, etc.) y reforzar el Made in Italy en cuanto a fortalecer las relaciones y la generación de conocimiento dentro de los distritos, la protección de los derechos de propiedad intelectual y la promoción del marketing territorial.

En este sentido, el futuro del distrito estará claramente ligado a la capacidad de reproducir y alimentar en el tiempo y hacia el exterior la compleja combinación de elementos que le ha permitido ser un modo alternativo de producción (de bienes y hoy cada vez más de servicios) y de aprendizaje técnico. Así, una política para el distrito deberá tener la capacidad de aprender de la experiencia, de involucrar a los principales protagonistas en la formulación y validación de los proyectos y generar una convergencia sobre los principios y estrategias en los ámbitos local, regional, nacional e internacional.

\section{Bibliografía}

Anatasia Bruno y Giuseppe Tattara (2003), Come mai il Veneto è diventato così ricco? Tempi, forme e ragioni dello sviluppo di una regione di successo, Universitá $\mathrm{Ca}^{\prime}$ Foscari, Venecia, Italia.

Anatasia Bruno y Giancarlo Corò (1996), Evoluzione di un'economia regionale. Il Nordest dopo il successo, Nuova Dimensione, Venecia-Portogruaro.

Antonelli, Cristiano (1999), Conoscenza tecnologica. Nuovi paradigmi dell'innovazione e specificità italiane, Edizioni della Fondazione Agnelli, Turín, Italia. 
Beccatini, Giacomo (1991), "Il distretto industriale marshalliano come concetto socio-economico", en Frank Pyke, Giacomo Becattini y Werner Sengerberger (eds.), Distretti industriali e corporazione fra le imprese, Studi e informazioni, Banca Toscana, Quaderni 34, Florencia, Italia, pp. 197-231.

Becattini, Giacomo (1998), Distretti Industriali e Made in Italy, Bolati-Boringhieri, Turín, Italia.

Becattini, Giacomo (2003), Luoghi, traslocali, benessere: Idee per un mondo migliore, Lezione, Bolonia, Italia.

Bellandi, Marco (1999), "Terza Italia e distretti industriali dopo la Seconda guerra mondiale", en Franco Amatori, Duccio Bigazzi, Renato Giannetti y Luciano Segreto (eds.), Storia d'Italia. L'Industria, Annali, 15, Einaudi, Turín, Italia, pp. 841-891.

Bertini, Silvano (1999), "Desarrollo endógeno de los sistemas locales de PyMEs. Lecciones desde la experiencia italiana”, mimeo.

Brusco, Sebastiano (1990), "The idea of the industrial district: its genesis”, en Frank Pyke, Giacomo Becattini y Werner Sengenberger (eds.), Industrial Districs and Inter-Firm, International Institute for Labour Studies, Génova, Italia, pp. 10-19.

Corò, Giancarlo (2001a), Processi produttivi, stato sociale, modelli di sviluppo, Ediesse, Venecia, Italia.

Corò, Giancarlo (2001b), "Servizi alle imprese e competitività del territorio", papeles de trabajo, Istituto di Scienze Economiche, Università di Urbino, Urbino.

Corò, Giancarlo (2001c), Quale politica industriale per i distretti in Veneto. Elementi di valutazione per la costruzione di un sistema di governance regionale, Università Ca'Foscari, Venecia, Italia.

Corò, Giancarlo (2002), "Le politiche peri distretti industriali da strumento 'speciale' a occasione per una nuova politi- 
ca economica regionale”, Economia e Società Regionale, 5, Ires Veneto, Milán, Italia, pp. 32-46.

Corò, Giancarlo (2003), “¿De anomalía a modelo de desarrollo? Prueba de madurez del noroeste italiano", Revista de Economía Exterior, 25, Madrid, España, pp. 55-71.

Corò Giancarlo y Mario Volpe (2003), "Frammentazione produttiva e apertura internazionale nei sistemi di piccola e media impresa", Economia e Società Regionale, 1, Ires Veneto, Milán, Italia, pp. 67-105.

Corò, Giancarlo y Stefano Micelli (2001), I distretti industriali nell' economia digitale: una convergenza possibile?, TEDISUniversità $\mathrm{Ca}^{\prime}$ Foscari, Venecia.

Dei-Ottati, Gaby (1997), "Cooperazione e concorrenza nel distretto industriale como modello organizzativo", en Luca Ferrucci y Riccardo Varaldo (eds.), Il distretto industriale tra logiche di impresa e logiche di sistema, Franco Angeli, Milán, Italia, pp. 85-95.

Grandinetti, Robeto y Raffaella Tabacco (2003), "I distretti industriali come laboratorio cognitivi”, mimeo.

Gurisatti, Paolo (2003), Una nuova dimensione e un nuovo paradigma per la Politica Industriale, Università Ca'Foscari, Venecia, Italia.

Krugman, Paul (1991), Geography and Trade, Leuven University-MIT Press, Boston, Estados Unidos.

Florida, Richard (2002), The Rise of the Creative Class: And How It's Transforming Work, Leisure, Community and Everyday Life, Basic Books, Nueva York, Estados Unidos.

Labory, Sandrine (2006), La internacionalización de los distritos en la Europa ampliada, Universidad de Ferrara, Ferrara, Italia.

Lane, David (2002), “Complexity and Local Interactions: Towards a Theory of Industrial Districts”, en Alberto Quadrio 
Curzio y Marco Fortis (eds.), Complexity and Industrial Clusters: Dynamics and Models in Theory and Practice, Physica-Verlag, Heidelberg-Nueva York, Estados Unidos, pp. 65-82.

Lanzetti, Renato y Roberto Marchionatti (2002), La Politica Industriale tra Europa e Regioni, Strument IRES, Piamonte, Italia.

Marshall, Alfred (1920), Principles of Economics, MacMillan, Londres, Inglaterra.

Marshall, Alfred y Mary Paley Marshall (1879), The Economics of Industry, MacMillan, Londres, Inglaterra.

Micelli, Stefano, María Chiaverso y Eleonora Di Maria (2003), "Processi di internazionalizzazione e strategie delle imprese distrettuali tra delocalizzazione e innovazione", Conferencia en el Istituto per il Commercio Estero "Internazionalizzazione e fragmmentazione della produzione nei distretti industriali”, Roma, Italia, 20-21 de marzo.

Micelli, Stefano, María Chiaverso y Eleonora Di Maria (2006a), "¿De las redes de pymes a los distritos virtuales? Evidencias sobre las recientes tendencias en Italia”, en Eleonora Di Maria y Stefano Micelli (comps.), Sistemas locales en las redes internacionales de producción, Venice International University-UNIDO, Roma, Italia, pp. 183-228.

Micelli, Stefano, María Chiaverso y Eleonora Di Maria (2006b), "Internacionalización de clusters y competitividad de pequeñas empresas", en Eleonora Di Maria y Stefano Micelli (comps.), Sistemas locales en las redes internacionales de producción, Venice International University-UNIDO, Roma, Italia, pp. 155-181.

Nonaka, Ikujiro e Hirotaka Takeuchi (1995), The knowledgecreating company, Oxford University Press, Nueva York, Estados Unidos.

Putnam, Robert, Robert Leopardi y Raffaella Nanetti (1993), Making democracy work. Civic traditions in Modern Italy, 
Princeton University Press, Princeton, Nueva Jersey, Estados Unidos.

Quadrio-Curzio Alberto y Marco Fortis (eds.) (2000), Il Made in Italy oltre il 2000, Il Mulino, Milán, Italia.

Rabelotti, Roberta (1997), External Economies and Cooperation in Industrial Distritics: A Comparison of Italy and Mexico, Macmillan, Londres, Inglaterra.

Saba, Andrea (1998), El modelo italiano: la especialización flexible y los distritos industriales, ILPES, Santiago de Chile.

Schmitz, Hubert (1999), "Efficienza collettiva e rendimenti di scala”, en Marco Di Tommaso y Roberta Rabellotti (eds.), Efficienza collettiva e sistemi di imprese, Il Mulino, Bolonia, Italia, pp. 23-99.

Tattara, Giuseppe (2003), "Il progresso tecnico nelle piccole imprese distrettuali”, mimeo.

Tattara, Giuseppe y Mario Volpe (2003), "Las redes en los distritos industriales italianos: la Terza Italia", en Fabio Boscherini, Marta Novick y Gabriel Yoguel (comps.), Nuevas tecnologías de información y comunicación. Los límites en la economía del conocimiento, Miño y Dávila-Ungs, Buenos Aires, Argentina.

Recibido: 7 de julio de 2006. Aceptado: 16 de octubre de 2006.

Rodrigo Reynaldo Carmona. Es candidato a doctor en ciencias sociales por la Facultad Latinoamericana de Ciencias Sociales (FLAcso, Buenos Aires); magíster en Strategie e Politiche di Sviluppo Locale e Distretti Industriali por la Scuola Superiore di Specializzazione/Fondazione Cassamarca, CEPAL (Conegliano-Treviso/Italia); magíster en economía y desarrollo industrial con especialización en pequeñas y medianas empresas por la Universidad Nacional de General Sarmiento (UNGS). Es miembro de la Red Nacional de Centros Académicos dedicados al Estudio de la Gestión en Gobierno Locales (Redmuni), miembro de la Red de Estudios sobre 
Pequeñas y Medianas Empresas (Redpymes-Mercour), miembro de la Comisión Directiva de la Asociación Latinoamericana para el Desarrollo de la Industria, la Tecnología y el Empleo (ALATIDE). Actualmente se desempeña como investigador-docente del Instituto del Conurbano (ICO) de la UNGS. Sus líneas de investigación actual son: políticas públicas, desarrollo local e institucional, governance y fomento productivo territorial. Entre sus publicaciones más recientes se encuentran: "Nuevas formas de gobierno y gestión pública en el escenario local. Algunos ejes de debate para el estímulo de procesos de desarrollo", en Adriana Rofman y Alejandro Villar (comps.), Desarrollo local. Una revisión crítica del debate, Espacio-UNQUI-UNGs, Buenos Aires, pp. 155-168 (2006); en coautoría, "Conocimiento y desarrollo local en el contexto global. Algunos debates en el escenario europeo", en Aída Quintar, José Borello, Verónica Roberts y Gabriel Yoguel (comps.) La informática en la Argentina. Desafíos a la especialización y a la competitividad, Prometeo-ungs, Buenos Aires, pp. 277-290 (2006); "Descentralización en la Provincia de Buenos Aires: una mirada desde los municipios", en Subsecretaría de Asuntos Municipales-Ministerio de Gobierno-Provincia de Buenos Aires (comps.), Descentralización y fortalecimiento del Estado, Gobierno de la Provincia de Buenos Aires, Buenos Aires, pp. 165-196 (2005). 